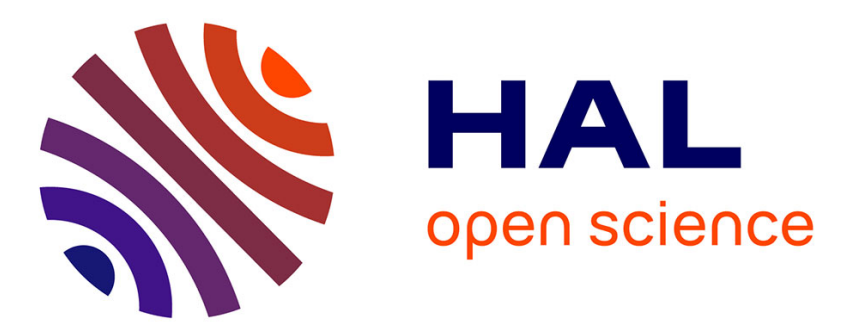

\title{
A Formalization of the French Elementary School Curricula
}

\author{
Oscar Rodríguez Rocha, Catherine Faron Zucker, Géraud Fokou Pelap
}

\section{To cite this version:}

Oscar Rodríguez Rocha, Catherine Faron Zucker, Géraud Fokou Pelap. A Formalization of the French Elementary School Curricula. Knowledge Engineering and Knowledge Management - EKAW 2016 Satellite Events, Revised Selected Papers, 2016, Bologne, Italy. hal-01619838

\section{HAL Id: hal-01619838 https://hal.inria.fr/hal-01619838}

Submitted on 9 Nov 2017

HAL is a multi-disciplinary open access archive for the deposit and dissemination of scientific research documents, whether they are published or not. The documents may come from teaching and research institutions in France or abroad, or from public or private research centers.
L'archive ouverte pluridisciplinaire HAL, est destinée au dépôt et à la diffusion de documents scientifiques de niveau recherche, publiés ou non, émanant des établissements d'enseignement et de recherche français ou étrangers, des laboratoires publics ou privés. 


\title{
A Formalization Of The French Elementary School Curricula
}

\author{
Oscar Rodríguez Rocha ${ }^{1}$, Catherine Faron Zucker $^{2}$, Geraud Fokou Pelap ${ }^{1}$ \\ 1 INRIA Sophia Antipolis Méditerranée, Sophia Antipolis, France. \\ (oscar.rodriguez-rocha, geraud.fokou-pelap)@inria.fr \\ 2 Univ. Nice Sophia Antipolis, CNRS, I3S, UMR 7271, Sophia Antipolis, France \\ faron@unice.fr
}

\begin{abstract}
In the education field, in order to achieve learning goals, it is necessary to define learning paths that foresee a gradual and incremental acquisition of certain knowledge and skills that students should acquire. In this paper we analyze the educational progressions of the French educational system, we show how to formalize them through a web ontology and how to perform knowledge extraction from the official texts describing them to automate the population of such an ontology.
\end{abstract}

Keywords: e-Education, Ontologies, Semantic Web, Knowledge Extraction, Knowledge Representation

\section{Introduction}

In the education field, in order to achieve learning goals, it is necessary to define learning paths that foresee a gradual and incremental acquisition of certain knowledge and skills that students should acquire. Such educational programs are usually defined by a body (a ministry or department of education) and then implemented by the schools or institutes that are part of the educational system. Nowadays it is not possible to find them in a standardized format that can be accessible and queried automatically.

In this context, we propose an answer to the following research questions:

1. How to model educational programs into a formal machine understandable ontology that can be processed and queried?

2. How to extract the knowledge and skills that an educational program requires from the official texts describing them?

3. How to automate the population of the ontology based on the extracted knowledge?

4. How can we exploit these formal representations?

Our scientific contribution is first, a web ontology that describes and represents an official standard of knowledge and skills, in this case the French educational system. It is formalized in the OWL Web Ontology Language, which enables to process and query it with SPARQL, the standard query language for 
the Web of data. Second, we propose a process to extract knowledge and skills from the official texts describing the French educational program. Finally, we propose a process to automatically populate our ontology with the knowledge extracted from the official texts which we further enrich with the web of data.

This work is a revised version of [10], published in the Proceedings of the Second International Workshop on Educational Knowledge Management co-located with the 20th International Conference on Knowledge Engineering and Knowledge Management (EKAW 2016)[8].

The remainder of this paper is structured as follows: Section 2 presents state of the art approaches of ontology-based modeling in e-Education. Section 3 introduces to the French common base of knowledge and skills for students as well as the official educational progressions. In Section 4 we propose EduProgression: an ontology to model the educational progressions of the French educational system. We show, in Section 5, how to exploit the data of the progressions modeled through EduProgression and its thesaurus. Section 6 presents our method to automate the population of this ontology by extracting elements of knowledge and skills from the official texts and Section 7 describes how to link them to relevant resources available on the Web of Data. Finally, the conclusions and future work are presented in Section 8.

\section{Related work}

The term of "educational ontology" covers very different types of ontologies, modeling different kinds of knowledge related to e-Education. In [11], the authors provide a literature survey of the development and use of ontologies in the domain of e-learning systems. According to the classification proposed in this survey, our work falls within the "curriculum modeling and management" category. Among such works we have CURONTO [3,4], an ontological model designed for the management of a curriculum and to facilite program review and assessment". Compared to it, our ontology is not focused on giving the means to conduct a review of the program nor an assessment; it focuses on the description and representation of the knowledge and skills required by an educational system and it provides the means to link knowledge and skills with resources from the web of data.

In the same category, Gescur [6] is a tool dedicated to the management and evaluation of the implementation of the curriculum, which facilitates the curriculum management process". It relies on an ontology of concepts relevant to curriculum management in Secondary Schools, such as teachers, departments, objectives, subjects, modules, tasks, documents, policies, activities, learning objects, quality criteria, etc. While this ontology focuses on the administration of the curriculum ours focuses on the management of the knowledge and skills required in each school year in the French educational system. In this sense, both ontologies could be complementary.

Besides the above cited literature review, the educational semantic web ontology proposed in [5] focuses on representing higher education concepts and 
assisting specialized e-learning systems. In contrast to this work, we propose an ontology that focuses on representing the elements of knowledge and skills of an educational system.

Finally, the OntoEdu educational platform architecture for e-Learning proposed in [7], relies on an activity ontology that describes all the education activities and the relations among them, and a material ontology which describes the educational content organization. The ontology is not available online, therefore it is not possible neither to analyze its classes and properties nor to determine the feasibility of use it in other scenarios outside the OntoEdu platform. Additionally their ontology does not contemplate the use of resources of the web of data to enrich the educational material.

\section{The French educational model}

In France, there is an official common base of knowledge and skills created by the Ministry of Education and published in the Official Journal; it is called the "Socle commun de connaissances et de compétences"3. It states that, compulsory education should at least guarantee to each student the necessary means to acquire a common base consisting of a set of knowledge and skills that are essential to master in order to successfully complete her education, build her personal and professional future and succeed in her life in society. The current common base of knowledge and skills entered into effect in 2016 while its predecesor leasted from 2006 to 2016. Our work was originally based on the first version of the French common base, but as we will show in this paper, it has been adapted to be compatible with the new version.

The French common base of 2006 was organized according to seven skills:

- Mastery of the French language

- Practice of a foreign language

- Basic math skills and scientific and technological culture

- Mastery of the common information and communication techniques

- Humanistic culture

- Social and civic competences

- Autonomy and initiative of the students

On the other hand, the current French common base is made up of five different skill domains:

- Language for communicate and thinking

- Methods and tools for studying

- Formation to the person and citizen

- Naturals systems and technical systems

- Representation of the world and human activities

${ }^{3}$ http://www . legifrance.gouv.fr/WAspad/UnTexteDeJorf?numjo=MENE0601554D 
These skill domains represent the knowledge and skills that students must acquire for a particular and essential requirement of the life in society.

In order to organize a gradual acquisition of the knowledge and skills of the French common base, some official learning progressions "Progressions pour l'ecole elmentaire" for the educational areas of the French elementary school have been defined and published (official bulletin of January 5, 2012). In the latest French common base, such progressions have been integrated implicitly into the division of the learning of each domain as three different cycles[2] with one precise goal for each of them. Thus, we have:

- Cycle 2 (CP-CE1-CE2): learning fundamentals

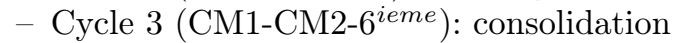

- Cycle 4 (5 $\left.5^{i e m e}-4^{i e m e}-3^{i e m e}\right)$ : deepening

These cycles were already defined in the common base of 2006 , but used for a different goal. For each elementary school cycle, the progressions are organized by area of education and they strictly follow the program labels to offer a comprehensive approach to knowledge, skills and attitudes to be mastered by the students. In this common base, the progressions are focused on a skill while in the common base of 2016 the progression are focused on a learning domain, that means on all the skills needed for a particular learning domain.

Our study is based on the analysis of these progressions (which are based on the French common base of knowledge and skills). By generalizing them, we built a standard model of the knowledge and skills necessary for the students through their educational path and a method to automate the population of this model by extracting knowledge from the texts describing these progressions. Being a standard model, it is also possible to model progressions from other educational systems in the world, such as the U.S. academic standards ${ }^{4}$ defined by the Common Core State Standards $(\mathrm{CCSS})^{5}$.

\section{The EduProgression ontology and associated thesaurus}

\subsection{The EduProgression ontology}

We created EduProgression, an ontological model formalized in the standard Ontology Web Language (OWL) for the formalization of educational progressions or programs as defined in the French common bases of 2006 [1] and 2016. In this formalization, we identified the following main classes:

- Element of Knowledge and Skills (EKS). As skills and knowledge are keystones of the common bases, this element is the key concept of our model. It is represented by the EKS class, which is the central class of the EduProgression ontology. This class is common for the two releases of the common base.

\footnotetext{
${ }^{4}$ http://www.corestandards.org/read-the-standards/

${ }^{5}$ http://www.corestandards.org/
} 
An element of knowledge and skills is associated to a set of knowledge pieces (class Knowledge) and/or skills (class Skill) for a specific French school cycle (class Cycle) or course (class Course) that may contain reference points (class PointOfReference) and also a vocabulary of associated terms (class Vocabulary). More precisely:

- Knowledge. Instances of class Knowledge are also instances of skos : Concept and each one belongs to a skos:ConceptScheme that contains all the knowledge pieces of a given progression. An instance of EKS is related to an instance of Knowledge through the property hasKnowledge.

- Skill. An instance of EKS is related to an instance of Skill through property hasSkill.

- Course. In the French common base of 2006, skills that students are expected to develop, are defined by cycle and each cycle is organized into courses. For example, "the consolidation cycle" includes "the second year elementary course" (CE2), "the first-year intermediate course" (CM1) and "the second year intermediate course" (CM2). In this context, an instance of class Course represents a course in a cycle. An instance of EKS is related to an instance of Course through property hasCourse.

- Cycle. In the French common base of 2016, skills that students are expected to develop, are defined only by cycle. An instance of EKS is related to an instance of Cycle through property hasCycle.

- PointOfReference. An instance of class PointOfReference represents an educational reference element on a specific element of knowledge and skills (an instance of EKS). An instance of EKS is related to an instance of class PointOfReference through property hasPointOfReference.

- VocabularyItem. Each element of knowledge and skills has vocabulary items. This vocabulary is compatible for the two common bases. An instance of EKS is related to an instance of class VocabularyItem through property hasVocabularyItem. A vocabulary item is also an instance of skos: Concept and it is related to an instance of skos:ConceptScheme which gathers the concepts of the thesaurus of the progression.

- Progression. In the common base released in 2006, the progressive acquisition of knowledge and skills is defined as a "progression", while for the current common base, the progressive acquisition of skills is defined as a "program". Therefore, a progression or a program in our model, is represented as an instance of the class Progression. It can be associated to an existing learning domain (through property hasLearningDomain) and to one or several EKSs (through property hasEKS).

- Learning domain. A learning domain represents a school subject like History or Mathematics. The learning domain is represented, in the ontology EduProgression, by an instance of the LearningDomain class, and it is also an instance of skos:Concept that is part of (only) one skos:ConceptScheme containing the only learning domains of a progression. Also, as they are SKOS concepts, learning domains are hierarchically organized by using the skos: broader and/or skos:narrower properties. A learning domain can be associated to a Progression or an EKS. 
- Skills Domain. For the common base of 2016, each domain of skills can be represented in our model by a class named SkillsDomain. An EKS can be associated to one or many skills domains through the property hasSkillsDomain to represent the skills domain(s) that it targets.

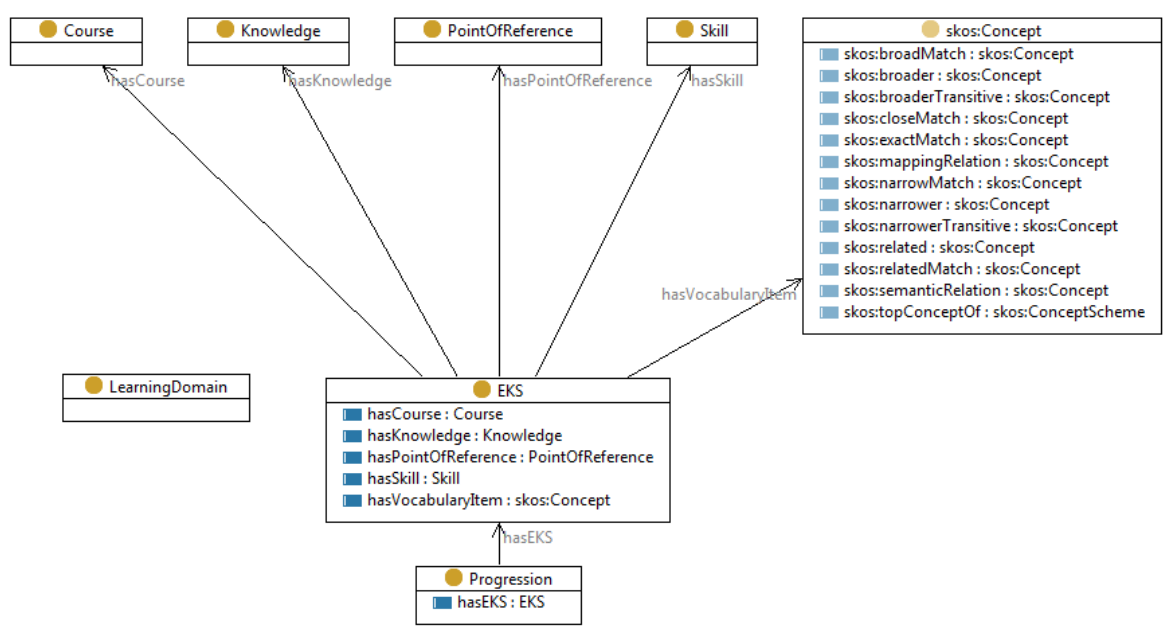

Fig. 1. Classes and Properties of the EduProgression Ontology

The EduProgression ontology is freely accessible online. ${ }^{6}$ It is composed of 7 OWL classes and 8 OWL object properties. The relationships between them can be seen in Figure 1. This figure presents the ontology for the education system released in 2006.

The two following excerpt is the description of the History progression and a related EKS representing the "european construction" based on EduProgression. We highlight with three different colors the three different types of resources related to specific thesaurus described in the following (see Section 4.2):

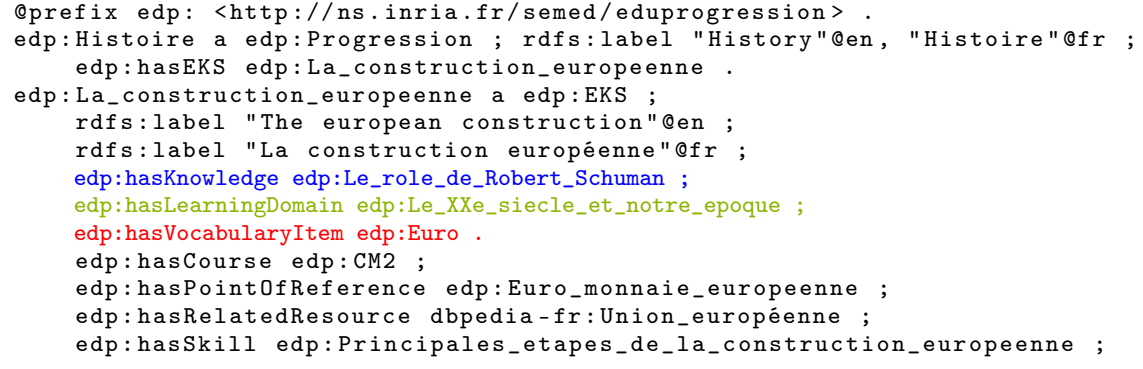

${ }^{6}$ http://ns.inria.fr/semed/eduprogression 


\subsection{Three thesaurus associated to each educational progression}

Each formalized progression is associated to three thesaurus: one for its knowledge pieces, one for its vocabulary items and another one containing its learning domains. As a result, knowledge pieces, vocabulary items and learning domains are represented by instance classes of the EduProgression ontology which also are SKOS concepts belonging to one of these thesaurus. Grouping these elements in thesaurus allows, among other benefits, to manage in a simple and structured way the knowledge of each progression [9].

The following excerpt shows the structure of the three thesaurus of the progression of History (in blue the thesaurus of knowledge pieces, in green the thesaurus of learning domains and in red the thesaurus of vocabulary items).

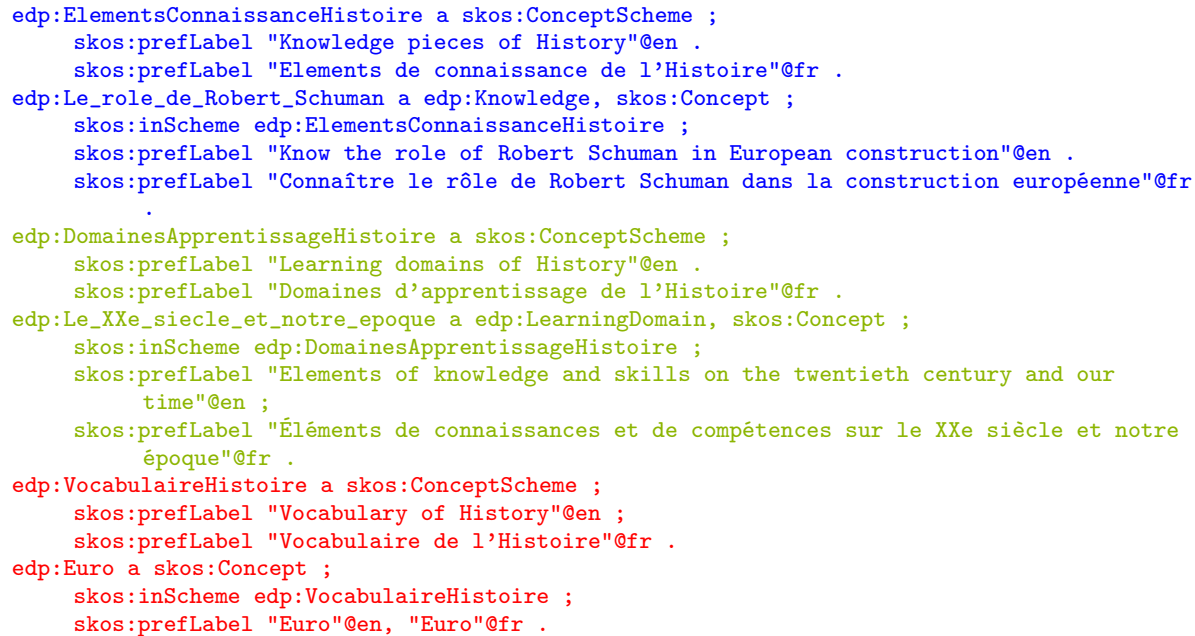

To give a clearer view of the elements of the History progression described in the previous excerpts, Figure 2 presents it as a graph.

At the time of writing, we have created the thesaurus of the vocabulary, knowledge pieces and learning domains relative to three progressions from the French curricula: History, Geography and Experimental sciences and technology, and we have populated the EduProgression ontology with the concepts in these thesaurus. For the progression on History, we identified 29 elements of knowledge and skills (instances of edp:EKS), the thesaurus of vocabulary items comprises 131 concepts, the thesaurus of knowledge pieces 45 concepts and the thesaurus of learning domains 7 concepts. For the progression of Geography, 32 elements of knowledge and skills were identified; its thesaurus of vocabulary items is composed of 131 concepts; its thesaurus of knowledge pieces and learning domains contaisn 34 and 6 concepts respectively. Finally, the pogression of Experimental sciences and technology was populated with a total of 50 elements of knowledge and skills; It has the largest vocabulary since it comprises 265 elements. Its thesaurus of knowledge pieces was populated with 40 concepts while its thesaurus of learning domains with 10 concepts. These thesaurus can be freely accessed online: http://ns.inria.fr/semed/eduprogression/thesaurus. 


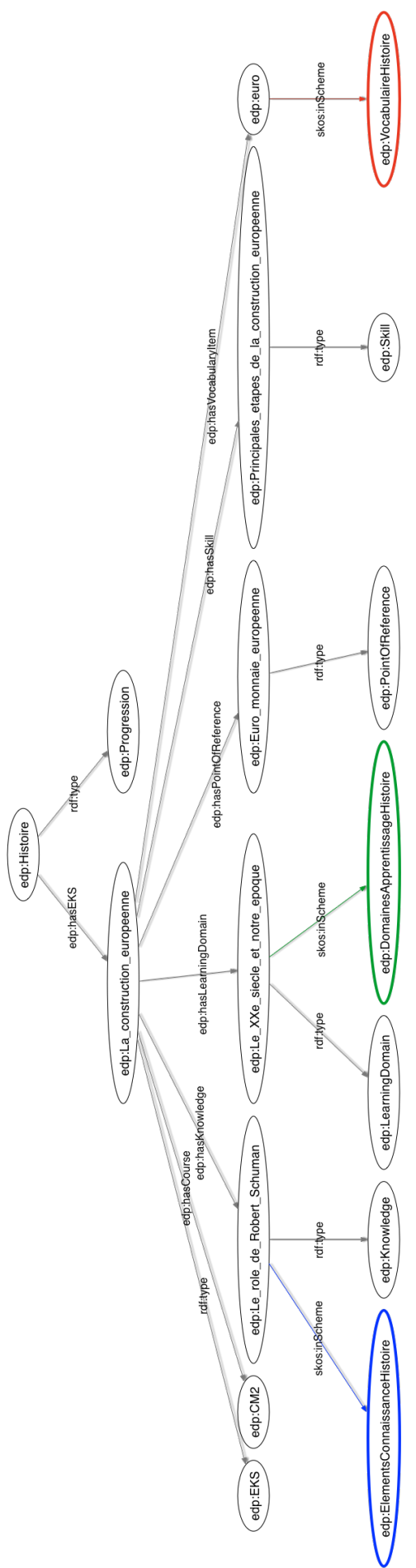

Fig. 2. Graph of the History progression excerpt 


\section{Exploitation of EduProgression and the associated thesaurus}

As the EduProgression ontology has been populated, we can exploit it with SPARQL queries. For example, to retrieve the $E K S$ required for the students attending the second year intermediate course (CM2) of History, we can use the following query:

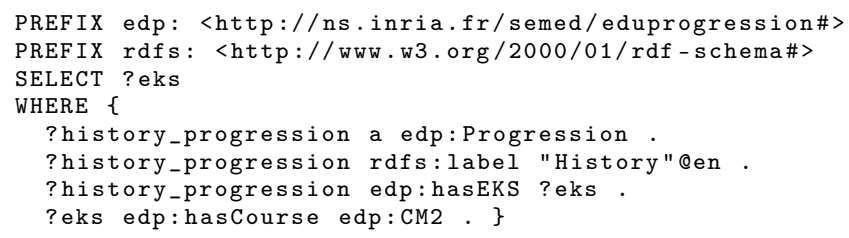

Similarly, we can retrieve the required vocabulary for the first-year intermediate course (CM1) of Geography, within the learning domain of "Elements of knowledge and skills on the territories at different scales".

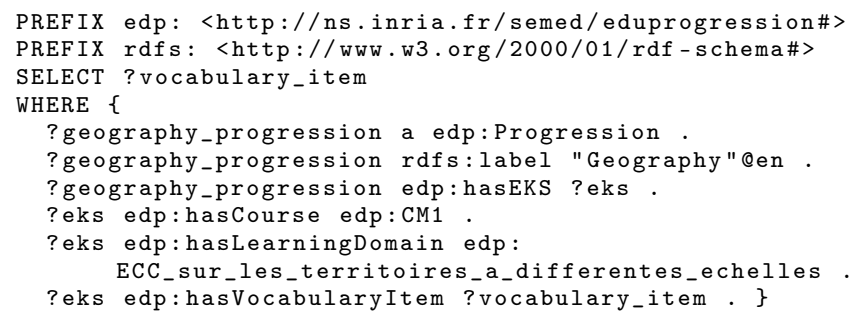

Finally, the following query allows to retrieve the necessary knowledge pieces of History and Experimental sciences and technology for the second year elementary course (CE2).

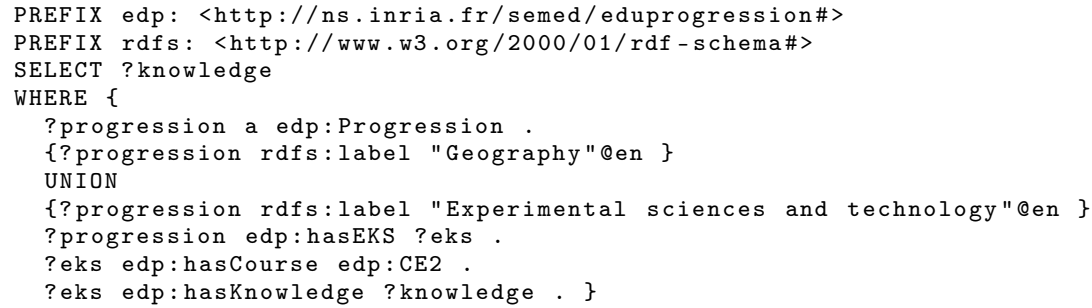

It is important to note that thanks to the EduProgression ontology it is possible to make queries to the existing progressions filtered by specific courses. This is the basis for the implementation of educational applications adapted to each student profile.

\section{Knowledge extraction from texts and formalization}

For the extraction of the information contained in the progressions for elementary school we had to consider first, that the progressions are only available in PDF 
format, that is, a PDF document for each learning domain. The data contained in each PDF, was inside tables without a standard format, therefore, when we tried to use common techniques for PDF data extraction ${ }^{7}$ such as OCR or data extraction from tables ${ }^{8}$, the resulting extracted text was mixed among various tables and/or incomplete.

This first led us to perform a preliminary manual extraction of knowledge from text. The first process that we experimented aimed at directly extracting RDF data from PDF texts. This involved to copy textual data directly from each PDF file, and then to paste it into an RDF description as literal values of some RDF triples (also manually written). Not only this process was time-consuming but also it was prone to human errors in the format of the data and the resulting RDF triples.

To solve these problems, we split the data extraction process in two steps, the first one consisting in manually filling a JSON intermediate data structure, the second one consisting in automatically transforming JSON data into RDF data. We designed an intermediate data structure in JSON format in which we represented each progression as a JSON object containing the following information:

- The name of the progression

- An array of the different learning sub domains involved in the progression

- A dictionary with the French scholar courses covered

- An array of the elements of knowledge and skills (EKS) involved in the progression. Each EKS itself contains:

- a title and a description in French

- the scholar cycle covered by this EKS

- the learning domain covered by this EKS

- a dictionary of the knowledge pieces (titles and descriptions in French) required by this EKS

- a dictionary containing the required skills (titles and descriptions in French) required by this EKS

- an array of the vocabulary items required by this EKS

- an array of points of reference required by this EKS

This allowed us to manually fill the data structure in a much faster and much more reliable way. In addition, in order to validate the format of the data, we defined a JSON schema ${ }^{9}$ representing these aforementioned structural constraints. This enabled us to automatically validated the JSON objects representing a progression manually entered.

As a second step, we defined and performed an automatic conversion process of JSON objects into RDF graphs. It uses the EduProgression ontology to automatically generate an RDF graph.

\footnotetext{
${ }^{7}$ https://en.wikipedia.org/wiki/Optical_character_recognition

8 http://tabula.technology/

${ }^{9}$ http://json-schema.org/
} 
The following excerpt is the intermediate JSON representation of the EKS on the Age of Discovery, which conversion in RDF is presented in the excerpt of section 4.2, which illustrates the use of the EduProgression ontology.

\section{$7 \quad$ Entity Linking}

We enriched the EduProgression ontology and its related thesaurus by conducting an entity linking process. It allows us to link the EKS instances of our already populated progressions, with DBpedia resources related to the textual values of the properties describing EKS instances. The information provided by these linked resources may be further exploited directly as learning material or to discover related learning resources.

To make this possible, we have created an automated process that first extracts literal values of the properties of each EKS and builds a textual representation of the latter by concatenating these literal values, and then retrieves the related DBpedia resources by using the DBpedia spotlight web service. The process is as follows:

1. All the instances of $E K S$ are retrieved from the populated progressions. This is the result of a SELECT SPARQL query processed by a SPARQL engine on our local triple store.

2. For each retrieved $E K S$ instance, the textual values of its properties are extracted and concatenated into a string which will be considered as a whole as the EKS text representation.

3. The process takes each EKS text representation and analyzes it by using the DBPedia Spotlight web service.

4. Each related resource obtained from the previous step is linked to the EKS by using the edp: hasRelatedResource property of EduProgression. This is done with an INSERT SPARQL query applied to our triple store.

The whole process is shown in Figure 3.

Currently, our process creates links between EKS instances and related resources in DBpedia. We chose to link EKS since they are conceived in our model as the main concept that represents skills and knowledge. Eventually, for other case studies, the EduProgression ontology could be adapted in such a way that the property hasRelatedResource can be applied to other types of instances, among which skills, knowledge pieces and vocabulary items, making it possible to link their instances with related resources from the web of data.

As a result of our process, we have obtained 187 resources related to the EKSs of the Geography progression with a precision of 94\%, 205 for the History progression with a precision of $95 \%$ and 220 for the progression of Experimental Sciences and technology with $94 \%$ of precision. For these experiments DBpedia was configured with a confidence of 0.15 and a support of 20. 


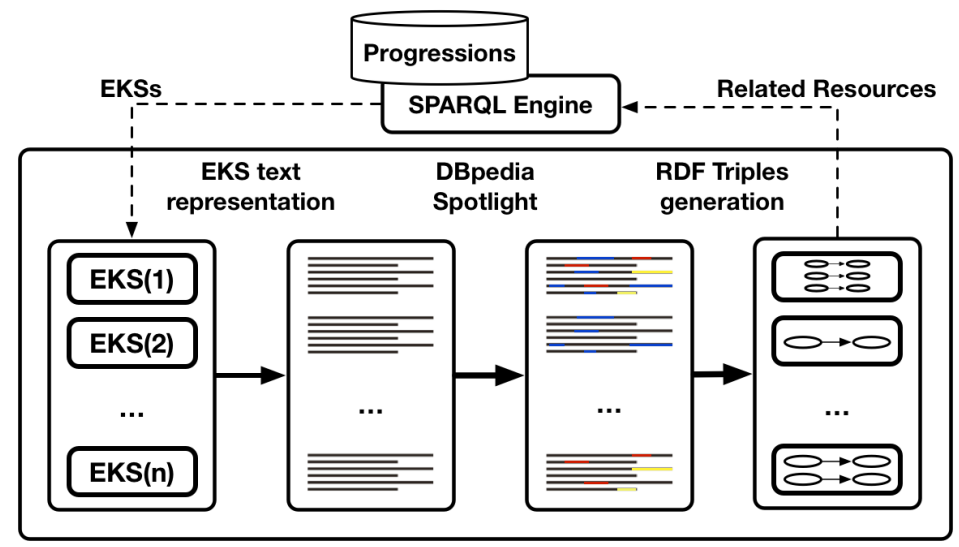

Fig. 3. Process to link EKS instances of a given progression to DBpedia resources

\section{Conclusions and future work}

In this paper, we have shown how to model an educational program by formalizing it as a Web ontology in OWL that is understandable by machines and that can be queried and processed. In particular, we have taken as a case study the French education system, from which we have extracted and formalized educational progressions in an ontology called EduProgression. We sketched how this ontology can be used through some example SPARQL queries. We have described a process for populating the EduProgression ontology, by extracting the knowledge elements and skills involved in the educational progressions from the official texts describing these progressions. Additionally, we showed how we managed to automatically link the ontology instances to Web resources.

As stated before, our work was originally based on the French "Socle commun de connaissances et de compétences" of 2006, however, as an update to this common base has been made in September of 2016, we have adapted our work accordingly, maintaining also the compatibility with the previous common base.

Future work includes the use of the EduProgression ontology and its related thesaurus for the indexation of educational resources to the French curricula through our ontology, with the aim of recommending them to students in their learning path. This activity will be performed in collaboration with our two industrial partners. In addition, we look forward to apply this work to other educational systems in the world as suggested in Section 3.

\section{References}

1. Le socle commun de connaissances et de compétences, http://media.education. gouv.fr/file/46/7/5467.pdf

2. Programmes d'enseignements, http://cache.media.education.gouv.fr/file/ MEN_SPE_11/67/3/2015_programmes_cycles234_4_12_ok_508673.pdf 
3. Al-Yahya, M., Al-Faries, A., George, R.: Curonto: An ontological model for curriculum representation. In: Proceedings of the 18th ACM Conference on Innovation and Technology in Computer Science Education. pp. 358-358. ITiCSE '13, ACM, New York, NY, USA (2013), http://doi.acm.org/10.1145/2462476.2465602

4. Alfaries, A., Al-Yahya, M., Chorfi, H., George, R.P.: Curonto: A semantic model of the curriculum for program assessment and improvement. INTERNATIONAL JOURNAL OF ENGINEERING EDUCATION 30(5), 1083-1094 (2014)

5. Bucos, M., Dragulescu, B., Veltan, M.: Designing a semantic web ontology for elearning in higher education. In: Electronics and Telecommunications (ISETC), 2010 9th International Symposium on. pp. 415-418 (Nov 2010)

6. Dexter, H., Davies, I.: An ontology-based curriculum knowledgebase for managing complexity and change. 2014 IEEE 14th International Conference on Advanced Learning Technologies 0, 136-140 (2009)

7. Guangzuo, C., Fei, C., Hu, C., Shufang, L.: Ontoedu: a case study of ontologybased education grid system for e-learning. In: GCCCE International conference, Hong Kong (2004)

8. Lahoud, I., Matta, N., Zablith, F., Ventura, S. (eds.): Proceedings of the Second International Workshop on Educational Knowledge Management co-located with 20th International Conference on Knowledge Engineering and Knowledge Management (EKAW 2016), Bologna, Italy, November 19-23, 2016, CEUR Workshop Proceedings, vol. 1780. CEUR-WS.org (2017), http://ceur-ws.org/Vol-1780

9. Pastor-Sánchez, J.A., Martínez Méndez, F.J., Rodríguez-Muñoz, J.V.: Advantages of thesaurus representation using the simple knowledge organization system (skos) compared with proposed alternatives. Information Research: An International Electronic Journal 14(4), n4 (2009)

10. Rocha, O.R., Faron-Zucker, C.: A formalization of standard knowledge and skills for the french elementary school curricula. In: Lahoud et al. [8], pp. 60-71, http: //ceur-ws.org/Vol-1780/paper6.pdf

11. Yahya, M.A., George, R., Alfaries, A.: Ontologies in e-learning: Review of the literature. International Journal of Software Engineering and Its Applications 9(2), 67-84 (2015) 